\title{
The Color Dependence of Interstellar Polarization
}

\author{
ALFRED BeHR \\ Göttingen University Observatory \\ Göttingen, Germany
}

$\mathbf{M}^{\mathrm{s}}$

EASUREMENTS OF THE INTERSTELLAR POLARIZATION of starlight at different wavelengths have been reported by several observers in references 1 to 8 . With a few exceptions the wavelength range of the measurements is still relatively small, and up to now only a few dozens of stars have been measured with high precision. There is still a remarkable discrepancy between the results of different observers, as can be seen in figure 1 . The scattering of the individual values is significantly larger than the probable error given by the respective authors. One of the most serious error sources seems to be the instrumental polarization which itself is highly color dependent. (See ref. 9.)

Nevertheless, even if a much greater individual error is assumed, a remarkable conclusion can be drawn: the wavelength dependence of polarization is not the same for all stars. If the Davis-Greenstein mechanism (ref. 10) is assumed as a working hypothesis, theoretical considerations presented in references 11 and 12 show that, in general, a maximum of polarization should be expected at a certain wavelength for a given type of interstellar dust. In this case any individual color dependence can be understood as a combined influence of more than one cloud with different types of interstellar grains, and, if necessary, with depolarization if the planes of vibration are not parallel to each other. However, it still seems to be impossible to draw conclusions from the observed color dependence about the type of material producing the polarization.

On the other side, according to reference 12 , a variation of the color dependence with the angle between the line of sight and the lines of magnetic force should be expected. In this instance a correlation with galactic longitude, or at least a significant difference between the Cygnus region and the Perseus region, should be observed, the effect of which, however, seems not to be very strongly indicated. Also, it is known that the small polarization in Cygnus is mostly due to depolarizing effects. 
There is still another way to discuss the observed results. According to reference 10 , the observed polarization $p$ as a function of the interstellar extinction $A_{V}$ and the angle $\sigma$ between the line of sight and of the lines of force is

$$
p / A_{V}=\left(p_{0} / A_{V}\right) \sin ^{2} \sigma
$$

where $p_{0}$ is the polarization for $\sigma=90^{\circ}$

Absence of depolarization indicates that the lines of force are in the plane of vibration. The observations reported in reference 4 show that the polarization can always be represented by the expression

$$
p / A_{\mathrm{v}} \leqq 0.065
$$

Small values should be observed when $\sigma<90^{\circ}$, or when depolarization occurs by different clouds with lines of force not in the same plane. For an individual star there is in general no possibility of deciding between the two cases. For large values of the ratio $p / A_{l}$, it can be easily assumed that $\sigma$ is not very much different from $90^{\circ}$.

For small values of the ratio $p / A_{l}$, a majority of stars with small values of $\sigma$ can be selected if stars with possible depolarization are excluded.

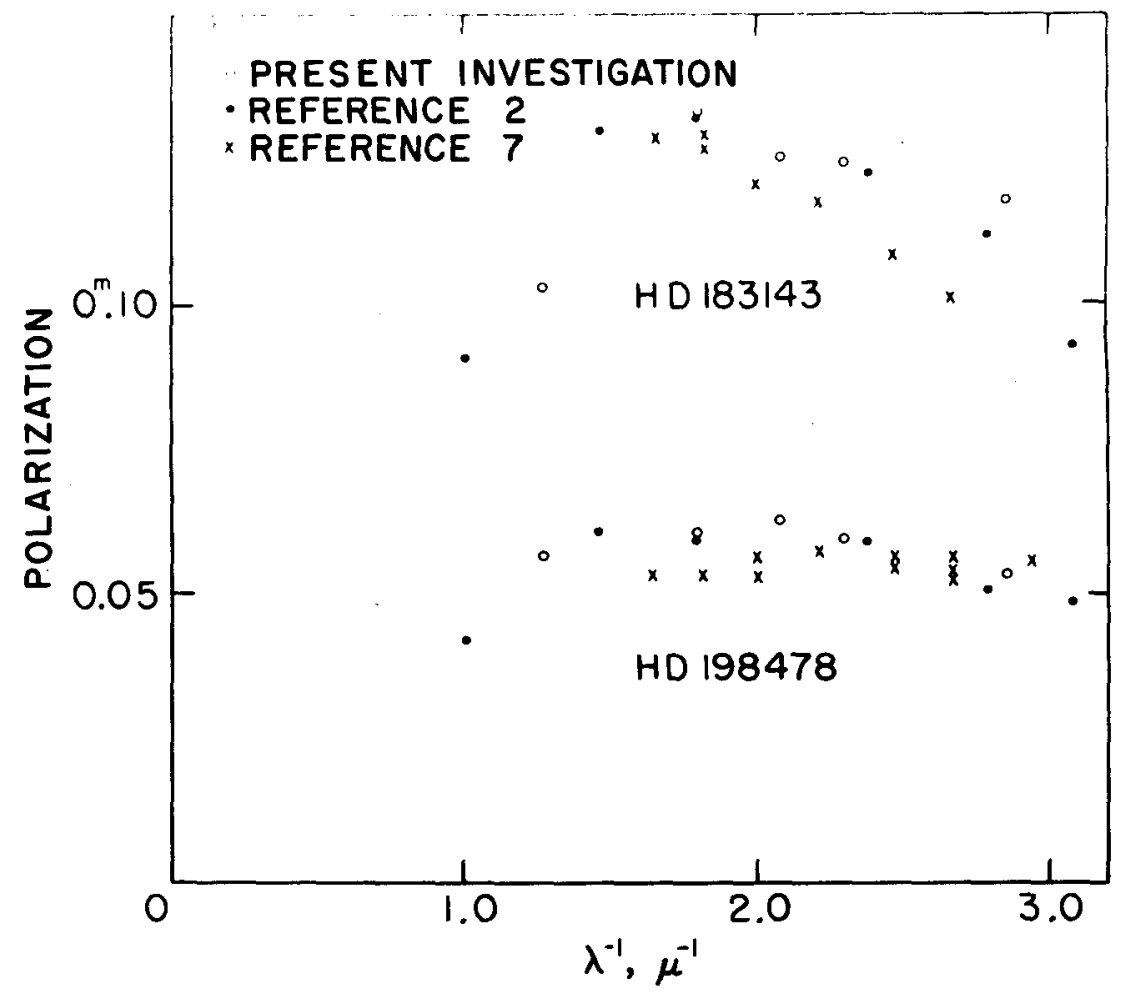

FIGURE 1. - Polarization as a function of wavelength for two stars. 


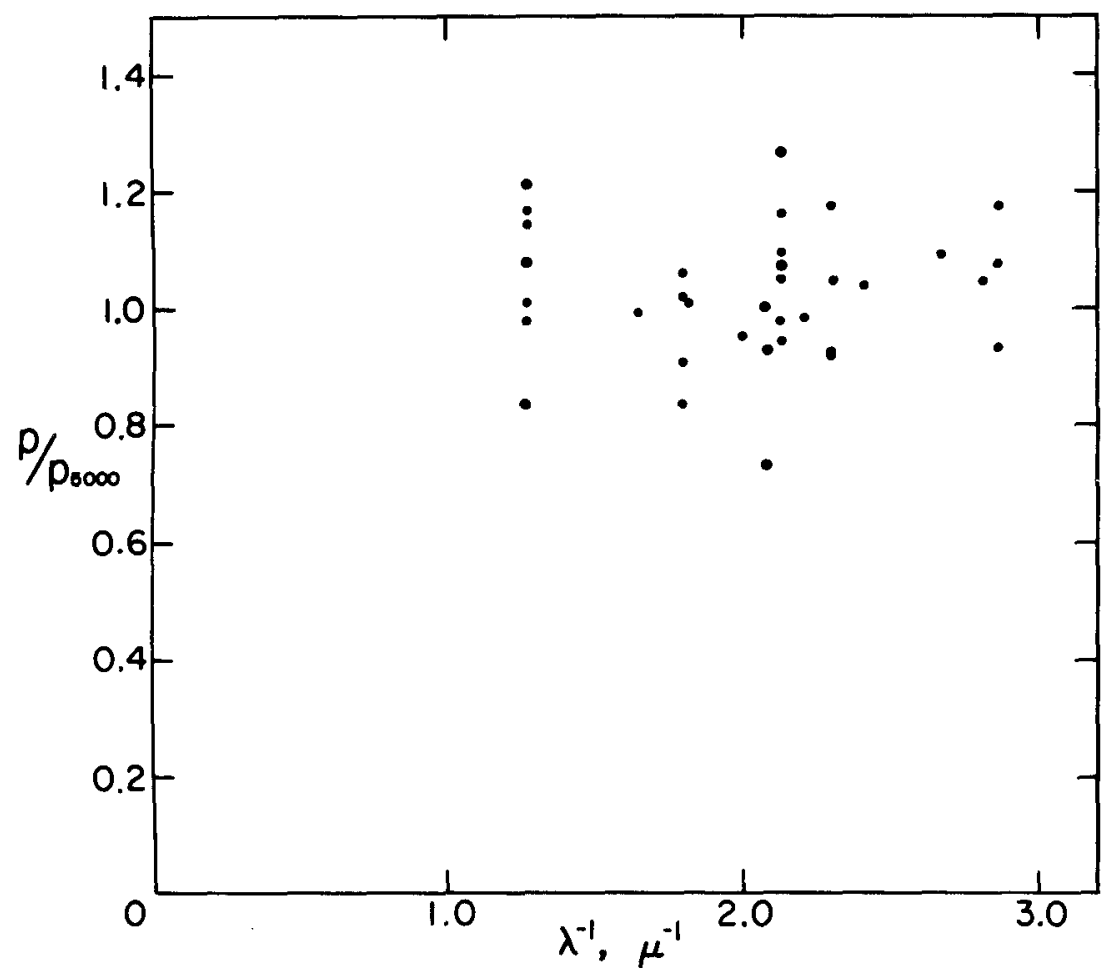

Figure 2. - Wavelength dependence of polarization for stars in group I.

This can be done if only polarizations with the plane of vibration parallel to the galactic equator are regarded. The material can therefore be divided into two groups:

group I: $p / A_{1} \leqq 0.027$ and $\theta= \pm 5^{\circ}$

group 11: all other stars

where $\theta$ is the angle between the plane of polarization and the galactic plane. In the first group are all stars with values of $\sigma$ very probably small, and in the second group are all stars with values of $\sigma$ certainly near $90^{\circ}$ for large values of the ratio $p / A_{V}$ and probably near $90^{\circ}$ for small values of the ratio $p / A_{V}$. due to depolarization. Although individual stars may deviate strongly, it can be concluded that the value of $\sigma$ is significantly smaller in group I than in group II. The result is shown in figures 2 and 3 , which contain results of references 2 and 7.

Figure 3 shows the color dependence as it was already known by Gehrels' measurements of six stars (all belonging to group II). Figure 2 does not show any significant color dependence, except perhaps for a weak minimum near $\lambda=5000 \AA$.

A comparison of these observations with results in the Orion aggregate obtained in 1965 by Appenzeller with the rotatable telescope at Yerkes Observatory in Williams Bay, Wisconsin, is shown in figure 4. 


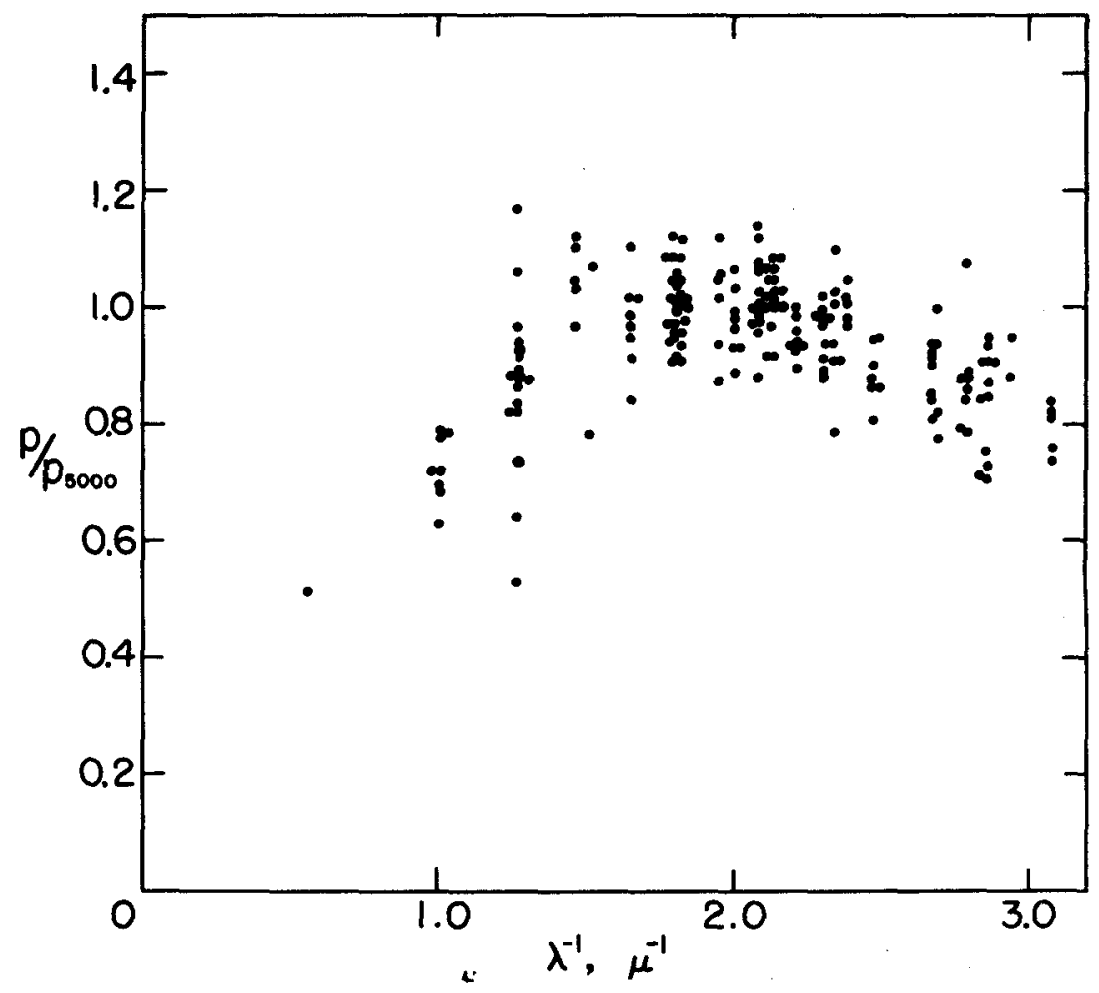

FIGURE 3. - Wavelength dependence of polarization for stars in group II.

Six Orion stars show a color dependence of polarization similar to that of group I; HD 43384, an Orion star in the background with larger polarization, HD 183143 in Sagitta used for comparison, and $\theta^{2}$ Ori exhibit a behavior like the stars in group II. The polarization of $\theta^{2}$ Ori may be influenced by the center of the bright Orion nebula.

In order to draw further conclusions from the results represented in figures 2 and 3 , it should be assumed that the value of $\sigma$ in the Orion aggregate, where the plane of vibration is nearly perpendicular to the galactic equator, is low, an assumption that seems to be in contradiction with the observations which yield a mean value for the ratio $p / 3 E_{B-V}$ of 0.040 , where $E$ is color excess. According to recent results (refs. 13 and 14), $A_{V} \approx 6 E_{B-V}$ in the Orion aggregate; therefore, $p / A_{V} \approx 0.020$, a result which admits a low value of $\sigma$. This new value of $p / A_{1}$ indicates that the plane of vibration does indeed appear perpendicular to the galactic equator.

\section{REFERENCES}

1. BEHR, A.: Beobachtungen zur Wellenlängenabhängigkejt der Interstellaren Polarisation. Zs. Astrophys., vol. 47, 1959, p. 54. 


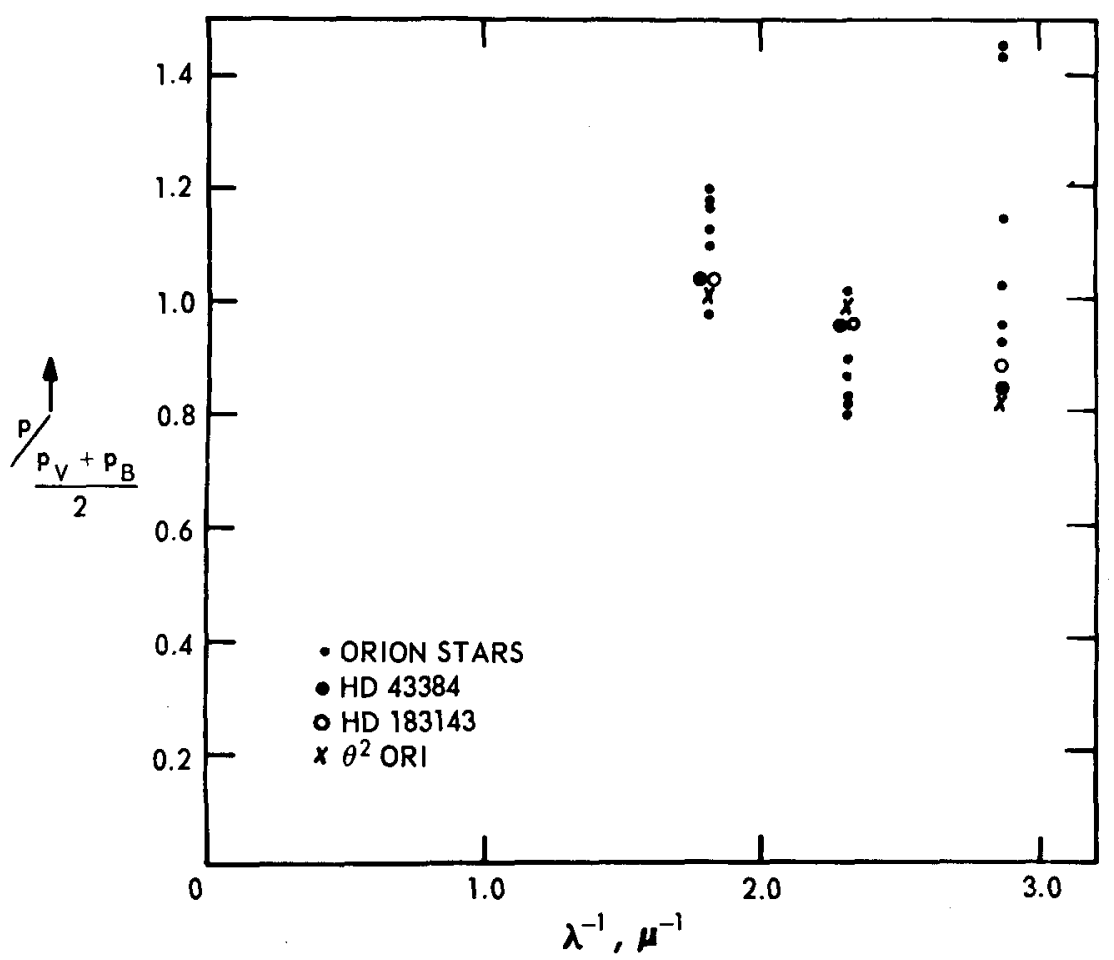

FIGURE 4.-Color dependence of six Orion stars. That of three other stars given for comparison.

2. Gehrels, T.: The Wavelength Dependence of Polarization Il. Interstellar Polarization. Astron. J., vol. 65, 1960, p. 470.

3. Hiltnen, W. A.: On Polarization of Radiation by Interstellar Medium. Phys. Rev., vol. 78, 1950, p. 170.

4. Hiltner, W. A.: Photoelectric, Polarization, and Spectrographic Observations of $O$ and B Stars. Astrophys. J., Suppl. Ser., vol. 2, 1956, p. 389.

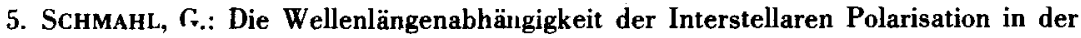
Umgebung der Assoziation VI Cygni. Zs. Astrophys., vol. 58, 1964, p. 165.

6. Serkowski, K.: Polarization of Galactic Clusters M25, NGC 869, 884, 1893, 2422, 6823, 6871, and Association VI Cygni. Astrophys. J., vol. 141, 1965, p. 1340.

7. Treanor, P. J.: Wavelength Dependence of Interstellar Polarization. Astron. J., vol. 68,1963 , p. 185.

8. Van den Bergh, S.: Polarisation des Sternlichtes im Gebiet der Plejaden. Zs. Astrophys., vol. 40, 1956, p. 249.

9. BEHR, A.: On Some Improvement in the Polarization Technique. Lowell Obs. Bull., vol. 4,1960 , p. 292.

10. Davis, L.; and Greenstein, J. L.: Polarization of Starlight by Aligned Dust Grains. Astrophys. J., vol. 114, 1951, p. 206.

11. Davis, L.: The Color Dependence of the Polarization of Starlight. Zs. Astrophys., vol. 47,1959 , p. 59.

12. Greenberg, J. M.; and Meltzer, A. S.: The Effect of Orientation of Non-Spherical Particles on Interstellar Extinction. Astrophys. J., vol. 132, 1960, p. 667. 
13. Johnson, H. L.; and Borgman, J.: The Law of Interstellar Extinction. Bull. Astron. Inst. Netherlands, vol. 17, 1963, p. 115.

14. Johnson, H. L.: Interstellar Extinction in the Galaxy. Astrophys. J., vol. 141, 1965, p. 923 .

\section{DISCUSSION}

Greenberg: The formula $p / A_{V}=\left(p_{0} / A_{V}\right) \sin ^{2} \sigma$ is derived on the basis of the Rayleigh approximation. Actually, however, this formula is not quantitatively correct.

Behr: Do you mean that this formula is completely wrong now?

Greenberg: It is restricted to Rayleigh particles - very small particles.

Wickramasinghe: It depends entirely on the sizes one uses. I feel that the formula holds for graphite grains with sizes of a few hundredths of a micron.

Greenberg: A spheroidal type of characteristics in the orientation effects must also be assumed.

Wickramasinghe: Yes, I am thinking of the case of anisotropic oblate spheroids. One can get this type of relation by using the Gans approximation. Of course, the Gans approximation may not be valid here.

Donn: In order to get a high extinction for these small particles, the Rayleigh-Gans approximation cannot be used because a small extinction would result in this instance.

Wickramasinghe: No, $I$ think in this instance that is not true. For small conducting particles the Rayleigh-Gans efficiency could get quite high.

Behr: If the angle $\sigma$ is zero, then polarization is zero too; and if $\sigma$ is $90^{\circ}$, then the polarization is a maximum; what happens between?

Greenberg: In between, the ratio of polarization to extinction doesn't follow $\sin ^{2} \sigma$.

Behr: Yes, I agree. I only assume this theory as a working hypothesis. A more correct one would probably change my separation limit of 0.027 . My only argument is that in group I the angle may be significantly smaller than in group II, not larger. This is what I emphasized in the paper. 